\title{
Simulations of Particle Dynamics in Magnetorheological Fluids
}

\author{
February 3, 1999
}

H.V. Ly ${ }^{1}$, F. Reitich ${ }^{2}$, M.R. Jolly ${ }^{3}$, H.T. Banks ${ }^{1}$, Kazi Ito ${ }^{1}$

\begin{abstract}
We present particle dynamics simulations for the response of magnetorheological (MR) fluids upon application of a magnetic field. The particles motion is considered to be governed by magnetic, hydrodynamic and repulsive interactions. Fluid-particle interactions are accounted for via Stokes' drag while inter-particle repulsions are modeled through approximate hard-sphere rejections. In accordance with their greater significance, on the other hand, (linear) magnetic interactions are fully simulated. The time evolution is considered to be magnetically quasi-static and magnetostatic forces are derived from the solution of (steady) Maxwell's equations, recomputed at each instant in time. For this we use a potential theoretic formulation where the boundary integral equations are solved with a fast multipole approach. We show that the resulting numerical codes can be effectively used to study a number of experimental observables such as effective magnetic permeabilities and response time-scales which are of crucial importance in the design of MR fluids.
\end{abstract}

AMS Subject Classification: 45L10, 65C20, 70F10, \& $78 A 35$

Keywords: Particle Dynamics, Fast Multipole Method, MR Fluids, Effective Permeability, Response Time Scale

\footnotetext{
${ }^{1}$ Center for Research in Scientific Computation, North Carolina State University, Raleigh, NC 27695-8205

${ }^{2}$ School of Mathematics, University of Minnesota, Minneapolis, MN 55455

${ }^{3}$ Material Division, Lord Corporation, 110 Lord Drive, Cary, NC 27511
} 


\section{Introduction}

Magnetorheological (MR) and electrorheological (ER) fluids constitute important classes of "smart" controllable materials. The essential characteristic of MR (resp., ER) fluids is that they may be reversibly transformed from a liquid state to that of a Bingham solid upon application of a magnetic (resp., electric) field. Although the discovery of these materials dates back to over half a century ago (Winslow [1947], [1949] for ER \& Rabinow [1948] for MR), their industrial realization had, until recently, proved elusive due, in part, to the stringent stability and durability requirements that such applications demand. Indeed, it was only a few years ago that commercially viable fluids of this type were achieved (Carlson et. al. [1990], [1994] \& Leventon [1993]) and controllable devices based on this technology are now beginning to evolve (see www.mrfluid.com). For the most part, these devices employ magnetic fluids as these present a number of advantages over their electric counterparts. These include higher achievable yield stresses (20 to 50 times those attainable with ER suspensions), lower voltage demands, a wider range of possible liquid carriers and lower sensitivity to contaminants and extremes in temperature (Weiss et al. [1992], [1993a], [1993b], Carlson at al. [1994], Jolly et. al. [1998a], [1998b]).

Typical ER and MR fluids consist of micron-sized electrically conducting or magnetically permeable particles dispersed in a carrier oil. It is this composition that, in fact, results in their field-dependent rheology: an applied field polarizes the particles which therefore move to reduce the stored electromagnetic energy of the ensemble. An energetically favorable arrangement consists of particle chains aligned in the direction of the applied field and this, in turn, gives rise to a strong resistance to applied strains (on the order of $100 \mathrm{kPa}$ for MR fluids), see e.g. Carlson et al. [1990], Weiss et al. [1992], Jolly et al. [1997], [1998a], [1998b]. In an attempt at understanding the main factors affecting this so-called "MR (or ER)-effect" and with a view to its possible enhancement for potential applications, a myriad of experimental and theoretical studies have been carried out since the effect was first recognized (see, e.g., Tao [1992], Bullough [1996] and references therein). The great number of variables that influence this behavior, however, conspired against the derivation of an accurate quantitative assessment of their relative importance since experiments could only explore small fractions of parameter space and theoretical work had to rely on oversimplified models. As it became practicable, on the other hand, it also became clear that numerical simulations on more complete models could deliver substantial information for the design of new or improved fluids. In particular, over the last decade, a number of numerical studies were performed on particle dynamics models for ER fluids that have sought to account for the different types of interactions (electric, hydrodynamic, Brownian, etc) that arise in the presence of applied fields (Klingenberg et al. [1989], Hass [1993], Hess and Weider [1996], Mohebi et al. [1996], Parthasarathy and Klingenberg [1996]). In this manner, the qualitative features of a number of experimental observables (such as the path of chain formation) were recreated and new insight was gained into the mechanisms responsible for the ER effect.

In order to make these models numerically tractable, a number of simplifications had to be performed. Some of them, such as neglecting Brownian forces, could be easily justified on the basis of simple scaling arguments (see Sec. 2.2). Others, however, were only made for

computational convenience. In fact, such was the case, for instance, with the inter-particle 
electromagnetic forces as well as with their hydrodynamic behavior. Although these are clearly the dominant effects within the system, their accurate mathematical representation required what appeared to be a "practically impossible" (Bonnecaze and Brady [p. 2188, 1991]) solution of Stokes or Maxwell's equations in a highly oscillatory geometry and were therefore, in all of these treatments, replaced by point-dipole and Stokes drag approximations respectively. We intend to show here, on the other hand, that some recent advances in the development of computational algorithms do indeed allow for an accurate and efficient treatment of such oscillatory problems. While it is still certainly true that standard finiteelement or boundary-element calculations on these models (where each particle, or particle boundary, would comprise several elements and where all of which would contribute to the overall field) are well beyond today's computational capabilities, we shall demonstrate that such calculations become practicable through the use of a fast multipole method (FMM).

The basic idea behind FMM (Rokhlin [1985] Greengard \& Rokhlin [1987] and Greengard [1988]) consists of using multipole expansions to calculate far-field contributions to a slowly decaying (e.g., Newtonian) potential at any given point. This observation can, in fact, be effectively used in a wide variety of (linear) potential calculations by appealing to the iterative solution of the corresponding boundary integral equations (see Sec. 3). For the present work we have initially implemented an FMM to handle the full (linear) electromagnetic interactions at each time step of a particle dynamics simulation (Sec. 3), while we have kept with previous models in treating the hydrodynamics via Stokes drag. We note however, that an analogous fast multipole approach can be used to solve the full Stokes equations of fluid flow (Greengard \& Kropinski [1999]). It should also be remarked that, in accordance with their greater practical importance, our interest lies with MR fluids which, in fact, we use for experimental validation (Sec. 4). While our model in its present state would, in principle, equally apply to ER fluids, we suspect that our assumptions on the dominance of electromagnetic and hydrodynamic forces constitutes a better approximation in the case of MR composites, as is evidenced by their aforementioned enhanced stability properties. On the other hand, a complete treatment of this magnetic case would necessitate the incorporation of nonlinear effects such as magnetic saturation. The means for such calculations will become clear, however, once we establish here the possibility of fully accounting for the linear magnetic interactions, as these calculations could be iterated to explore the nonlinear regime. In any case, these considerations and those pertaining to other possible extensions (Stokes equations, three-dimensional geometries, models of rheological response, other higher order effects, etc) will be left for future work.

\section{Equations of Motion}

\subsection{Governing Forces and Equations}

In this section, we derive the equations of motion for circular particles in $\mathbb{R}^{2}$ in the presence of an external magnetic field $\overrightarrow{\mathbf{H}}_{0}$. The motion of the kth particle is described by Newton's second law of motion

$$
\mathcal{M}_{k} \frac{d^{2} \overrightarrow{\mathbf{x}}_{k}}{d t^{2}}=\overrightarrow{\mathbf{F}}_{k}
$$


subject to the initial position and velocity

$$
\overrightarrow{\mathbf{x}}_{k}^{0}=\overrightarrow{\mathbf{x}}_{k}(0) \quad \text { and } \quad \overrightarrow{\mathbf{v}}_{k}^{0}=\overrightarrow{\mathbf{v}}_{k}(0),
$$

where $\mathcal{M}_{k}$ is the mass, $\overrightarrow{\mathbf{x}}_{k}$ is the center, and $\overrightarrow{\mathbf{F}}_{k}$ is the total force acting on the kth particle, which occupies the region $\Omega_{k}$. Contributions to $\overrightarrow{\mathbf{F}}_{k}$ arise from several sources, namely (see e.g., Klingenberg et. al. [1989], Parthasarathy \& Klingenberg [1996] and Mohebi, Jamasbi \& Liu [1996]):

\section{- Magnetic Forces}

The magnetic force on $\Omega_{k}$ can be calculated from the local field $\overrightarrow{\mathbf{H}}$ with the aid of the Maxwell stress tensor $\sigma^{\mathrm{Max}}=\mu_{k} \mu_{0}\left[\overrightarrow{\mathbf{H}} \overrightarrow{\mathbf{H}}^{\mathcal{T}}-\frac{1}{2}|\overrightarrow{\mathbf{H}}|^{2} \delta\right],\left(\delta=\right.$ unit tensor, $\mu_{k}, \mu_{0}=$ the permeability for the kth particle and the carrier oil, respectively) as

$$
\overrightarrow{\mathbf{F}}_{k}^{\operatorname{mag}}=\frac{1}{2 \pi \mu_{0}} \int_{\partial \Omega_{k}} \sigma^{\mathrm{Max}} \cdot \vec{n} d S,
$$

where $\overrightarrow{\mathbf{n}}$ is the unit normal vector on $\partial \Omega_{k}$.

\section{- Hydrodynamic Forces}

For small Reynolds number, the hydrodynamics can be decoupled from the magnetostatic problem and can be approximated by the Stokes' drag (see Batchelor [1967] pp. 244 for details). Indeed, the hydrodynamic force can be approximated using Oseen's equations for flow due to a moving body at small Reynolds number as

$$
\overrightarrow{\mathbf{F}}_{k}^{\mathrm{hydro}}=-D \frac{d \overrightarrow{\mathbf{x}}_{k}}{d t} .
$$

For circular cylinders for instance, the Stokes drag coefficient $D$ is given by $D=\frac{4 \pi \eta_{c}}{\log \frac{7.4}{R e}}$ per unit length. Here $\eta_{c}$ is the carrier oil absolute viscosity and Re is the Reynolds number. Formulation (2.4) has been widely used to approximate the hydrodynamic force in dynamic simulations for both MR and ER fluids (see, e.g., Klingenberg et. al. [1989], Hass [1993] and Mohebi, Jamasbi \& Liu [1996]).

\section{- Repelling Forces}

In our simulations, we shall assume that both the particles and the container walls are "hard". To approximate this regime, we shall follow the work of Klingenberg et. al. [1989] and Mohebi et. al. [1996] and propose that a "repelling force" acts on the kth particle as it approaches others or a wall of the container. A simple model for such a force is given, for instance, by

$$
\overrightarrow{\mathbf{F}}_{k}^{\mathrm{rep}}=-\mu_{k} H_{0}^{2} R \sum_{l=1}^{M} \overrightarrow{\mathbf{r}}_{k l} e^{-\beta\left|d_{k l}\right|}-\overrightarrow{\mathbf{n}}_{k} \mu_{k} H_{0}^{2} R e^{-\beta\left|d_{k}^{\text {wall }}\right|},
$$

where $M$ is the total number of particles, $H_{0}$ is the intensity of the applied magnetic field, $R$ is the radius of the particle, $\beta>0$ is the repelling parameter, $\overrightarrow{\mathbf{r}}_{k l}=\frac{\overrightarrow{\mathbf{x}}_{l}-\overrightarrow{\mathbf{x}}_{k}}{\left|\overrightarrow{\mathbf{x}}_{l}-\overrightarrow{\mathbf{x}}_{k}\right|}$, $d_{k l}=\operatorname{dist}\left(\Omega_{k}, \Omega_{l}\right)$. The wall repelling force uses $\overrightarrow{\mathbf{n}}_{k}$, an outward unit normal vector at a point $p$ on the boundary of the container $\Omega$ where $p$ is closest to $\overrightarrow{\mathbf{x}}_{k}$ on $\partial \Omega$, and $\left|d_{k}^{\text {wall }}\right|=\operatorname{dist}\left(\Omega_{k}, \partial \Omega\right)$. Other models, including polynomial forms, have been studied by Klingenberg et. al. [1989]. 


\subsection{Dimensional Analysis}

Our simulations will proceed on the non-dimensionalized equations of motion which we now derive. Let us begin by denoting the dimensionless variables with an asterisk and performing the following scalings in equations (2.1)-(2.2)

$$
\begin{array}{ll}
\overrightarrow{\mathbf{x}}=R \overrightarrow{\mathbf{x}}^{*}, & \\
t=\tau t^{*} & \tau \text { is the scaling time, } \\
\overrightarrow{\mathbf{F}}_{k}^{\mathrm{mag}}=F_{k}^{s} \overrightarrow{\mathbf{F}}_{k}^{\text {mag, },}, & F_{k}^{s}=\mu_{k} H_{0}^{2} R, \\
\overrightarrow{\mathbf{F}}_{k}^{\mathrm{rep}}=F_{k}^{s} \overrightarrow{\mathbf{F}}_{k}^{\mathrm{rep}, *}, & \overrightarrow{\mathbf{F}}_{k}^{\mathrm{rep}, *}=-\sum_{l=1}^{M} \overrightarrow{\mathbf{r}}_{k l} e^{-\beta\left|d_{k l}\right|}-\overrightarrow{\mathbf{n}}_{k} e^{-\beta\left|d_{k}^{\text {wall }}\right|,} \\
\overrightarrow{\mathbf{F}}_{k}^{\text {hydro }}=-\frac{D R}{\tau} \frac{d \overrightarrow{\mathbf{x}}_{k}^{*}}{d t^{*}}, & \tau=\frac{D R}{F_{k}^{s}}=\frac{4 \pi}{\mu_{k} \log \frac{7.4}{R e} \frac{\eta_{c}}{H_{0}^{2}} .}
\end{array}
$$

It then follows that

$$
\mathcal{G}_{k} \frac{d^{2} \overrightarrow{\mathbf{x}}_{k}^{*}}{d t^{* 2}}=-\frac{d \overrightarrow{\mathbf{x}}_{k}^{*}}{d t^{*}}+\overrightarrow{\mathbf{F}}_{k}^{\mathrm{mag}, *}+\overrightarrow{\mathbf{F}}_{k}^{\mathrm{rep}, *}
$$

where

$$
\mathcal{G}_{k}=\frac{\mathcal{M}_{k} R}{F_{k}^{s} \tau^{2}}=\frac{\rho_{p} R^{2} H_{0}^{2} \mu_{k}}{32 \pi^{2}\left[\log \frac{7.4}{R e}\right]^{2} \eta_{c}^{2}}
$$

is a dimensionless constant and $\rho_{p}$ is the density of the particle per unit length. For the MR fluids we seek to model, the particles are basically composed of iron with diameters of 3 to 5 microns and $\mu_{k} \cong 10^{3} \times \mu_{0}$. The carrier oil viscosity $\eta_{c} \cong 0.01-0.1 \mathrm{~Pa}$-s and the applied magnetic flux $\mu_{0} H_{0} \cong 0.001-0.1$ Tesla, so that $\mathcal{G}_{k} \cong 10^{-4}$. Thus the term on the left of (2.6) is several orders of magnitude smaller than those on the right hand side, and we therefore set it to zero. Consequently, the equations of motion (2.1)-(2.2) become

$$
\frac{d \overrightarrow{\mathbf{x}}_{k}^{*}}{d t^{*}}=\overrightarrow{\mathbf{F}}_{k}^{\mathrm{mag}, *}+\overrightarrow{\mathbf{F}}_{k}^{\mathrm{rep}, *} \quad \text { and } \quad \overrightarrow{\mathbf{x}}_{k}^{0}=\overrightarrow{\mathbf{x}}_{k}(0)
$$

It is to be remarked that for MR fluids with characteristics as described above, the thermal effects from the continuous phase molecules on the particles are quite small for rapid field application (Mohebi et. al. [1996]). Therefore, the magnetostatic forces largely dominate the Brownian forces. More precisely, the ratio of the Brownian force to the magnetic force is approximately given by $\lambda=\frac{2 \pi \mathcal{K} T}{R^{3} \mu_{k} H_{0}^{2}}$, where $\mathcal{K}=1.38 \times 10^{-23}$ Joules $/ \mathrm{K}$ is the Boltzmann's constant and $T=298 \mathrm{~K}$ is the operating temperature. In our case, this ratio is of order $10^{-8}$ which justifies our neglecting the effects of Brownian motion.

\section{Magnetic Forces}

Clearly, the main challenge with the model (2.7) consists of the calculation of the highly oscillatory magnetic interactions $\left\{\overrightarrow{\mathbf{F}}_{k}^{\mathrm{mag}, *}\right\}_{k=1}^{M}$. Indeed, an accurate estimation of such forces demands the continuous knowledge of the local magnetic field. $\overrightarrow{\mathbf{H}}$ as the particles rearrange themselves, so that Maxwell's equations must be resolved at each instant in time. More precisely, let us consider $\Omega \subset \mathbb{R}^{2}$ which is filled with a non-magnetic viscous fluid and $M$ permeable circular particles $\Omega_{1}, \Omega_{2}, \cdots, \Omega_{M}$. Then, since the electromagnetic time scale 
is much shorter than that of the motion itself, we may safely assume that the fields are governed by the equations of magnetostatics, namely,

$$
\nabla \cdot \overrightarrow{\mathbf{B}}=0, \quad \text { and } \quad \nabla \times \overrightarrow{\mathbf{H}}=\overrightarrow{\mathbf{J}},
$$

where $\overrightarrow{\mathbf{J}}$ is the free current, $\overrightarrow{\mathbf{H}}$ is the magnetic field and $\overrightarrow{\mathbf{B}}$ is the magnetic induction. In our case, the material constituents are isotropic and hence

$$
\overrightarrow{\mathbf{B}}=\overrightarrow{\mathbf{B}}_{i}+\mu_{0} \overrightarrow{\mathbf{H}}=\mu \overrightarrow{\mathbf{H}},
$$

where $\overrightarrow{\mathbf{B}}_{i}$ is the intrinsic induction caused by magnetization, $\mu_{0}$ is the permeability of free space and

$$
\mu= \begin{cases}\mu_{k} & \text { in the kth particle } \\ \mu_{0} & \text { in the carrier oil. }\end{cases}
$$

Notice that $\left\{\mu_{k}\right\}_{k=1}^{M}$ are not necessarily identical and are substantially larger than $\mu_{0}$, $\left(\mu_{k} \sim 2000 \mu_{0}\right)$. In general, $\mu_{k}$ is defined as a function of $\overrightarrow{\mathbf{H}}$ to model magnetic saturation. For moderate fields, however, $\mu_{k}$ may be accurately approximated as a constant and we shall take this as our working assumption. Moreover, for the composites we consider, no free currents are present in the domain. Thus the second equation in (3.1) becomes $\nabla \times \overrightarrow{\mathbf{H}}=0$ which, in turn, implies that the magnetic field can be written in terms of a scalar potential $\phi$,

$$
\overrightarrow{\mathbf{H}}=-\nabla \phi .
$$

As a result, equations (3.1)-(3.4) can be simplified to

$$
\nabla \cdot(\mu \nabla \phi)=0
$$

with $\mu$ given by (3.3) and $\mu_{k}, \mu_{0}$ constant. Note that (3.3) implies that equation (3.5) has highly oscillatory coefficients. Also, of course, equation (3.5) encodes the continuity of the magnetic potential $\phi$ and of the normal component of $\overrightarrow{\mathbf{B}}$. That is, for any $k=1,2, \ldots, M$,

$$
\begin{array}{ccc}
\lim _{\substack{p \rightarrow \partial \Omega_{k} \\
p \in \Omega_{k}}} \phi(p) & =\lim _{\substack{p \rightarrow \partial \Omega_{k} \\
p \in \Omega_{k}^{\mathrm{c}}}} \phi(p) \\
\lim _{\substack{p \rightarrow \partial \Omega_{k} \\
p \in \Omega_{k}}} \mu_{k} \frac{\partial \phi}{\partial \vec{n}_{k, p}}(p)= & \lim _{\substack{p \rightarrow \partial \Omega_{k} \\
p \in \Omega_{k}^{\mathrm{c}}}} \mu_{0} \frac{\partial \phi}{\partial \vec{n}_{k, p}}(p)
\end{array}
$$

where $\vec{n}_{k, p}$ is an outward unit normal vector at $p \in \partial \Omega_{k}$ and $\Omega_{k}^{\mathrm{C}}=\Omega \backslash \bar{\Omega}_{k}$ is the complement of $\Omega_{k}$.

\subsection{Integral Equation Formulation and Boundary Element Discretization}

Although, as we said, the coefficients of equation (3.5) are rapidly changing in space, they do remain constant in each $\Omega_{k}$. Thus the overall potential can be derived from appropriate charge densities supported on the boundaries of the particles. These densities satisfy certain 
integral equations which are, in principle, amenable to solution by finite (boundary) element approximation. As we shall discuss below (Sec. 3.2), the difficulties associated with the high computational cost of classical boundary element approximation for this kind of problem can, in fact, be overcome through the implementation of the Fast Multipole Method.

To derive the integral equations, let us denote by $\partial \Omega_{0}$ the boundary of the domain $\Omega$ and impose the following Dirichlet boundary condition on $\partial \Omega_{0}$

$$
\left.\phi\right|_{\partial \Omega_{0}}=f
$$

A potential $\phi$ satisfying (3.5)-(3.8) can be represented by single-layer and double-layer integrals, see, e.g., Colton \& Kress [1983] and Greengard \& Moura [1994], in the form

$$
\phi(p)=\sum_{j=1}^{M} \int_{\partial \Omega_{j}} G(p, q) \eta_{j}(q) d s(q)+\int_{\partial \Omega_{0}} \frac{\partial G}{\partial \vec{n}_{\partial \Omega_{0}, q}}(p, q) \xi(q) d s(q), \quad p \in \Omega .
$$

Here, $\vec{n}_{\partial \Omega_{0}, q}$ is the outward unit normal vectors at $q \in \partial \Omega_{0}$ and $G(p, q)=\frac{1}{2 \pi} \log |p-q|$ is the fundamental solution of the Laplace's equation in $\mathbb{R}{ }^{2}$. The functions $\eta_{j}$ 's on $\left\{\partial \Omega_{j}\right\}_{j=1}^{j=M}$ and $\xi$ on $\partial \Omega_{0}$ are appropriate (unknown) surface densities. Note that the potential $\phi$ in (3.9) automatically satisfies

$$
\Delta \phi=0 \quad \text { on } \Omega_{k} \quad \text { for } k=0,1, \ldots, M,
$$

and the continuity condition (3.6) at the interfaces. In this regard, we remark here that our representation of the potential $\phi$ differs from the standard one described in most of the pertinent literature. Indeed, while a standard representation would involve both single- and double-layer potentials at each material interface, the formulation (3.9) implicitly guarantees the continuity of the potential at particle-fluid boundaries. In addition, the use of a sole double-layer integral on the exterior boundary ensures that the equations for $\eta_{j}$ 's and $\xi$ that result from (3.7)-(3.8) constitute a system of Fredholm equation of the second kind. In fact, using the jump relations of potential theory (see, e.g., Jaswon [1977] and Colton \& Kress [1983]), we obtain from equations (3.7)-(3.8) the following system of integral equations,

$$
\begin{gathered}
\eta_{k}(p)-2 \lambda_{k} \sum_{j=1}^{M} \int_{\partial \Omega_{j}} \frac{\partial}{\partial \vec{n}_{k, p}} G(p, q) \eta_{j}(q) d s(q) \\
-2 \lambda_{k} \frac{\partial}{\partial \vec{n}_{k, p}} \int_{\partial \Omega_{0}} \frac{\partial G}{\partial \vec{n}_{\partial \Omega_{0}, q}}(p, q) \xi(q) d s(q)=0, \\
\xi(p)+2 \sum_{j=1}^{M} \int_{\partial \Omega_{j}} G(p, q) \eta_{j}(q) d s(q)+2 \int_{\partial \Omega_{0}} \frac{\partial G}{\partial \vec{n}_{\partial \Omega_{0}, q}}(p, q) \xi(q) d s(q)=2 f(p),
\end{gathered}
$$

where $\lambda_{k}=\frac{\mu_{k}-\mu_{0}}{\mu_{k}+\mu_{0}}$ and equations (3.10)-(3.11) hold for $p \in\left\{\partial \Omega_{k}\right\}_{k=1}^{M}$ and $\partial \Omega_{0}$, respectively.

Our approach to the solution of equations (3.10)-(3.11) (at any fixed instant in time) relies on the inversion of their discretized version. To this end, we divide each boundary $\left\{\Omega_{j}\right\}_{j=0}^{M}$, including the exterior boundary, into $N_{j}$ disjoint elements $\left\{\gamma_{j}^{l}\right\}_{l=1}^{N_{j}}$ and we denote 
by $\left\{p_{j}^{l}\right\}_{l=1}^{N_{j}}$ the midpoint of $\left\{\gamma_{j}^{l}\right\}_{l=1}^{N_{j}}$ for $j=0,1, \cdots, M$ and $N=\sum_{j=0}^{M} N_{j}$. To derive the approximate equations, we assume that the unknown densities $\left\{\eta_{j}\right\}_{j=1}^{M}$ and $\xi$ in (3.10)-(3.11) are constant over each element, with collocation taken at the midpoints. That is,

$$
\int_{\gamma_{j}^{l}} U(p, q) \varrho(q) d s(q) \cong \varrho\left(p_{j}^{l}\right) \int_{\gamma_{j}^{l}} U(p, q) d s(q)
$$

for $\rho$ being either $\eta_{j}$ or $\xi$ and $U$ being one of the kernels in (3.10)-(3.11). Thus, denoting $\eta_{j}^{l}=\eta_{j}\left(p_{j}^{l}\right)$ and $\xi^{l}=\xi\left(p_{0}^{l}\right)$, equations (3.10)-(3.11) can be transformed into a matrix equation

$$
\left(\mathbf{I}+\mathbf{M}_{N}\right) \mathbf{U}_{N}=\mathbf{F}_{N} .
$$

Here, $\mathbf{I}$ is the $N \times N$ identity matrix,

$$
\mathbf{M}_{N}=\left[\begin{array}{ll}
\mathbf{A} & \mathbf{B} \\
\mathbf{C} & \mathbf{D}
\end{array}\right], \quad \mathbf{U}_{N}=\left[\begin{array}{l}
\Phi \\
\vec{\xi}
\end{array}\right], \quad \mathbf{F}_{N}^{i}= \begin{cases}0 & 1 \leq i \leq \tilde{N} \\
2 f\left(p_{0}^{i}\right) & \tilde{N}+1 \leq i \leq \tilde{N}+N_{0},\end{cases}
$$

where $\tilde{N}$ denotes $\sum_{j=1}^{M} N_{j}$,

$$
\begin{aligned}
& \Phi=\left[\begin{array}{c}
\vec{\eta}_{1} \\
\vec{\eta}_{2} \\
\vdots \\
\vec{\eta}_{M}
\end{array}\right], \quad \vec{\eta}_{k}=\left[\begin{array}{c}
\eta_{k}^{1} \\
\eta_{k}^{2} \\
\vdots \\
\eta_{k}^{N_{k}}
\end{array}\right] \text { for } 1 \leq k \leq M, \quad \vec{\xi}=\left[\begin{array}{c}
\xi_{1} \\
\xi_{2} \\
\vdots \\
\xi_{N_{0}}
\end{array}\right] \\
& \mathbf{A} \Phi\left(p_{k}^{i}\right)=-\frac{\lambda_{k}}{\pi} \sum_{j=1}^{M} \sum_{l=1}^{N_{j}} \eta_{j}^{l} \int_{\gamma_{j}^{l}} \frac{\left(p_{k}^{i}-q\right) \cdot \vec{n}_{k, p_{k}^{i}}}{\left|p_{k}^{i}-q\right|^{2}} d s(q), \\
& \mathbf{B} \vec{\xi}\left(p_{k}^{i}\right)=-\frac{\lambda_{k}}{\pi} \sum_{l=1}^{N_{0}} \xi_{0}^{l} \int_{\gamma_{0}^{l}} \frac{\partial^{2}}{\partial \vec{n}_{k, p_{k}^{i}} \partial \vec{n}_{l, q}} \log \left|p_{k}^{i}-q\right| d s(q), \\
& \mathbf{C} \Phi\left(p_{0}^{i}\right)=\frac{1}{\pi} \sum_{j=1}^{M} \sum_{l=1}^{N_{j}} \eta_{j}^{l} \int_{\gamma_{j}^{l}} \log \left|p_{0}^{i}-q\right| d s(q), \\
& \mathbf{D} \vec{\xi}\left(p_{0}^{i}\right)=\frac{1}{\pi} \sum_{l=1}^{N_{0}} \xi_{0}^{l} \int_{\gamma_{0}^{l}} \frac{\left(q-p_{0}^{i}\right) \cdot \vec{n}_{\gamma_{0}^{l}, q}}{\left|p_{0}^{i}-q\right|^{2}} d s(q) .
\end{aligned}
$$

\subsection{Numerical Implementation}

To solve for $\mathbf{U}_{N}$ in (3.12), we use an iterative method, namely GMRES (Generalized Minimal RESidue). As an iterative solver, GMRES demands the repeated calculation of products $\left(\mathbf{I}+\mathbf{M}_{N}\right) \mathbf{U}_{N}$. The matrix $\mathbf{M}_{N}$ in our linear system (3.12) is fully dense so that multiplication of $\mathbf{M}_{N}$ and $\mathbf{U}_{N}$ would require $\mathcal{O}\left(N^{2}\right)$ operations. However, as we describe below, by exploiting the physical nature of the underlying magnetostatic problem it is possible to accurately approximate these matrix vector multiplication by a procedure, the Fast Multipole Method (FMM), whose operation count is only of $\mathcal{O}(N \log N)$. 
The FMM algorithm was introduced in Rokhlin [1985] for rapid solution of integral equations in potential theory and later extended by Greengard \& Rokhlin [1987] to fast evaluation of Coulombic interactions in large-scale systems of particles. A typical calculation of the field at a given point due to a distribution of $N$ charges $\left(\mathbf{M}_{N} \mathbf{U}_{N}\right.$, in our notation) can be broken into two parts, the far field and the near field calculations. However, since the Coulombic potential decays rather slowly (logarithmically), the far-field calculation must, in principle, account for all interactions among the particles and, consequently, the number of operations for calculating the field is $\mathcal{O}\left(N^{2}\right)$. The goal of the fast multipole method is to reduce this number of operations while accounting for all interactions and maintaining any desirable order of accuracy. To achieve this, the basic strategy consists of clustering the charges at different spatial lengths to allow for the computation of the interactions between clusters by using multipole expansions. Near-field interactions are computed directly. This systematic process is the key ingredient in reducing the number of operations in the numerical matrix-vector multiplications. Complete details of the FMM algorithm can be found in Greengard [1988]. For other applications and implementations of the FMM see also Rokhlin [1985] [1990], Greengard \& Rokhlin [1987] [1989], Greengard \& Gropp [1990], Greengard \& Strain [1990], Engheta et. al. [1992], Murphy et. al. [1993], Greengard \& Moura [1994], Jones et. al. [1994], McKenney et. al. [1995], and Greengard \& Kropinski [1999].

\subsection{Error Analysis}

In this subsection, we present a simple error analysis for the solution of the boundary element method discussed in the previous subsection. Since we employ the midpoint rule for all the quadratures, the order of convergence is expected and will be shown to be at least quadratic. Let us start with a system of integral equations $(\mathbf{I}+\mathbf{M}) \mathbf{U}=\mathbf{F}$ where $\mathbf{U}, \mathbf{F} \in L^{2}(\Gamma)$ and $\mathbf{M}: L^{2}(\Gamma) \longrightarrow L^{2}(\Gamma)$ is a compact operator (the $L^{2}(\Gamma)$-topology can, of course, be substituted by others depending on the regularity of $\mathbf{U})$. We assume $\mathbf{M}$ takes the following form

$$
\mathbf{M U}(p)=\int_{\Gamma} K(p, q) U(q) d q .
$$

Also, the discretized boundary integral equations can be written as an $N \times N$ system

$$
\left(I+M_{N}\right) U_{N}=F_{N}
$$

with the midpoint collocated right-hand side $\left[F_{N}\right]_{i}=F\left(p_{i}\right)$ for $1 \leq i \leq N$, where the $p_{i}$ 's are the midpoints of the segments $\left\{\Gamma_{i}\right\}_{i=1}^{N}$, so that $[(I+M) U]\left(p_{i}\right)-\left(I+M_{N}\right) U_{N}=0$. By defining $\left(P_{N} U\right)_{i}=U\left(p_{i}\right) \chi_{\left\{p_{i}\right\}}$, we have

$$
\left(I+M_{N}\right)\left(U_{N}-P_{N} U\right)=P_{N} M U-M_{N} P_{N} U .
$$

For sufficiently smooth boundaries and $M$ compact, it can be shown that (see Kress [1989])

$$
\left\|\left(I+M_{N}\right)^{-1}\right\|_{\left\{l^{2} ; l^{2}\right\}} \leq \mathcal{K}, \quad \text { independently of } N .
$$

It follows from (3.17) and (3.18) that

$$
\left\|U_{N}-P_{N} U\right\|_{l^{2}} \leq C_{1} \mathcal{K}\left\|P_{N} M U-M_{N} P_{N} U\right\|_{l^{2}}
$$


for $N>0$. Let $E=P_{N} M U-M_{N} P_{N} U$ denote the locally truncated error at $p$. Since we are using the midpoint quadrature rule, for $p$ away from the midpoints

$$
|E(p)|_{l^{2}}=\left|\int_{\Gamma} K(p, q) U(q) d q-\sum_{i=1}^{N} K\left(p, p_{i}\right) U\left(p_{i}\right)\right| \Gamma_{i}||_{l^{2}} \leq \mathcal{O}\left(N^{-2}\right),
$$

which implies

$$
\left\|U_{N}-P_{N} U\right\|_{l^{2}} \leq C_{2} \mathcal{K} N^{-2} .
$$

For $p=p_{i}$, we compute $\int_{\Gamma_{i}} K\left(p_{i}, q\right) U(q) d q$ exactly (see Greengard and Moura [1994]).

\section{Numerical Results}

In this section, we first describe the physical regime and parameters for the dynamic simulations of five MR fluids. We then discuss our results and quantify the magnetic responses based on their time scales and effective permeabilities.

\subsection{Microstructure Evolutions in MR Fluids}

The dimensions for the rectangular domain that we consider for dynamic simulation are $L_{x} \times L_{y}=0.237 \mathrm{~mm} \times 0.1 \mathrm{~mm}$. We assume that all particles are circular, initially centered randomly at $\left\{\overrightarrow{\mathbf{x}}_{i}\right\}_{i=1}^{M}$. We consider five samples of MR fluids, corresponding to $5 \%, 10 \%$, $15 \%, 20 \%$ and $30 \%$ volume fractions. There are 170, 341, 511, 682, and 1024 particles for the respective samples. In addition, we assume the following physical parameters in our simulations:

$$
\begin{array}{ll}
\text { Radius } & R=1.5 \text { micrometers } \\
\text { Fluid Density } & \rho_{0}=10^{3} \mathrm{~kg} / \mathrm{m}^{3} \\
\text { Particle Density } & \rho=7 \times \rho_{0} \\
\text { Fluid Permeability } & \mu_{0}=4 \pi 10^{-7} N / A^{2} \\
\text { Particle Permeability } & \mu_{k}=2000 \times \mu_{0} . \\
\text { Viscosity } & \eta_{c}=0.1 \mathrm{~Pa} \mathrm{~s} \\
\text { Applied Field } & H_{0}=7.96 E 4 \mathrm{~A} / \mathrm{m}\left(\mu_{0} H_{0}=0.10 \text { Tesla }\right) .
\end{array}
$$

The repelling parameter in (2.5) and the Reynolds number are assumed to be 40 and 0.01 , respectively. For the boundary conditions on the rectangle, we assume $\phi$ satisfies

$$
\phi(x, y)=\left\{\begin{array}{cc}
H_{0} L_{y} & 0 \leq x \leq L_{x}, y=0 \\
H_{0} L_{y}\left(1-\frac{y}{L_{y}}\right) & 0 \leq y \leq L_{y}, x=L_{x} \\
0 & 0 \leq x \leq L_{x}, y=L_{y} \\
H_{0} L_{y}\left(1-\frac{y}{L_{y}}\right) & 0 \leq y \leq L_{y}, x=0 .
\end{array}\right.
$$

The results for the dynamic simulations for $10 \%, 20 \%$ and $30 \%$ volume fraction MR fluids are displayed in Figures 1-3 respectively. 

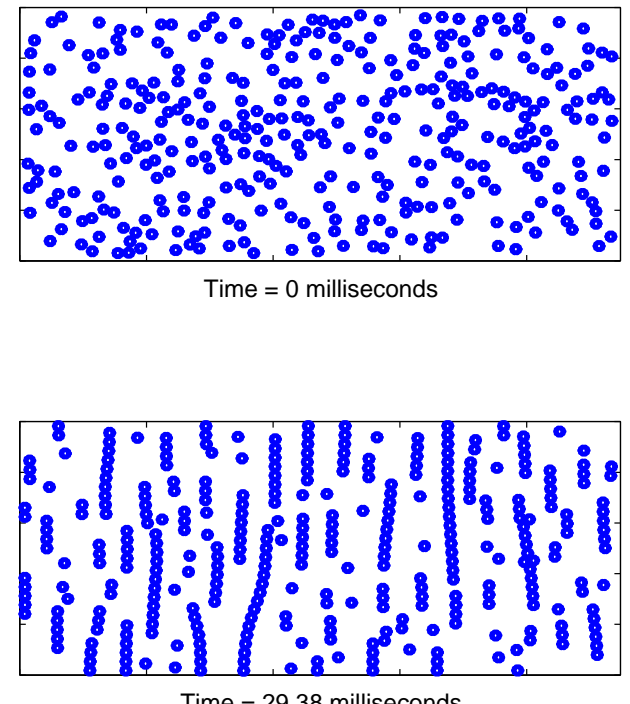

Time $=29.38$ milliseconds

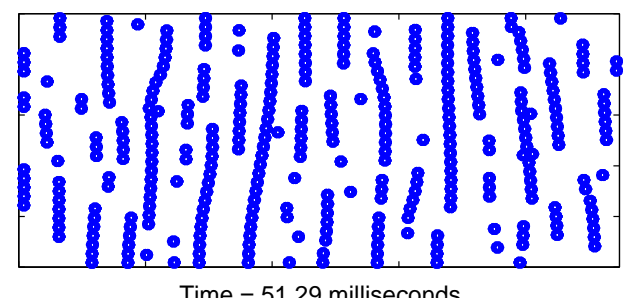

Time $=51.29$ milliseconds
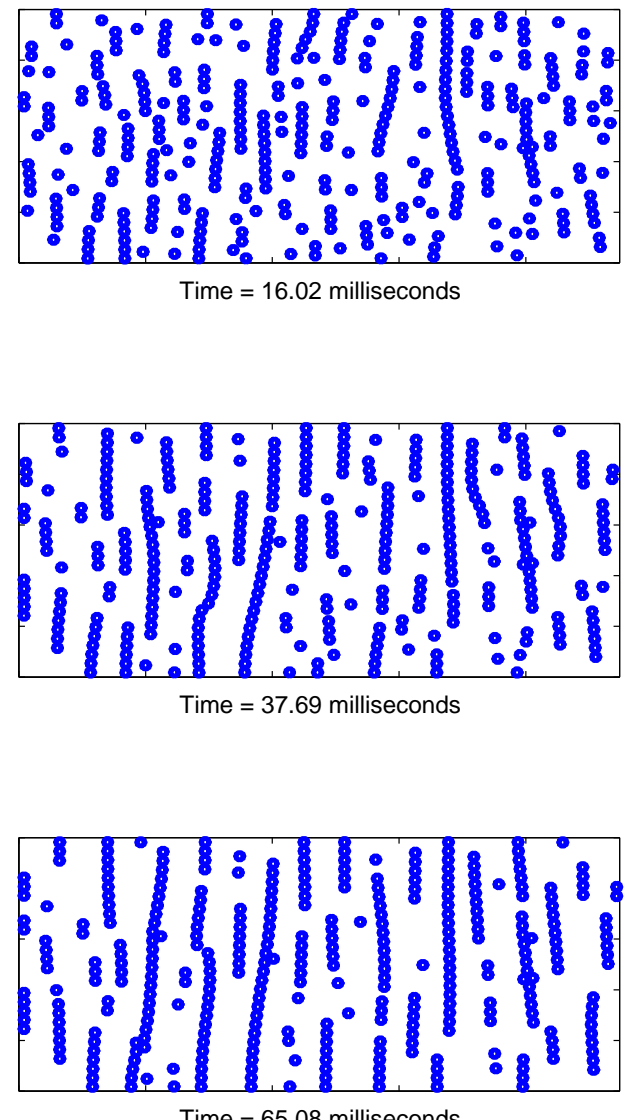

Time $=65.08$ milliseconds

Figure 1: dynamic simulations for MR fluid with $10 \%$ volume fraction.

Based on these results, we observe that, at first, particles form short fragmented chains in the direction of the applied field. Subsequently these short chains merge together and form longer chains. As time progresses further, these chain-like clusters continue to lengthen, align and approach a steady state. We also observe that the time for the columnar structures to form and the particle volume fraction $(\varphi)$ of the sample are inversely related.

\subsection{Quantifications of Microstructure Evolutions}

Recent experimental work has been conducted to indirectly measure the microstructure response in MR fluids using real-time permeability measurements (Jolly et. al [1997]) and analogously in ER fluids using permitivity measurements (Blackwood et. al. [1994]). In both cases, experimental data were fitted with exponential functions in an attempt to identify the time constants $t_{1}$ for microstructure formation. A theoretical estimate of such constants can be easily derived by consideration of the time response of a pair of point 


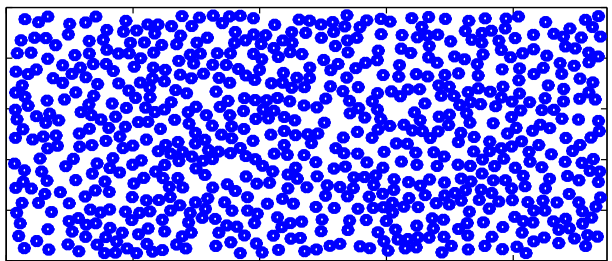

Time $=0$ milliseconds

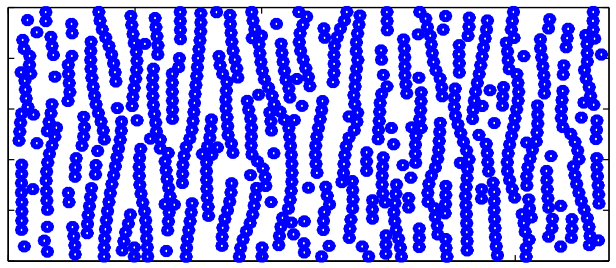

Time $=7.059$ milliseconds

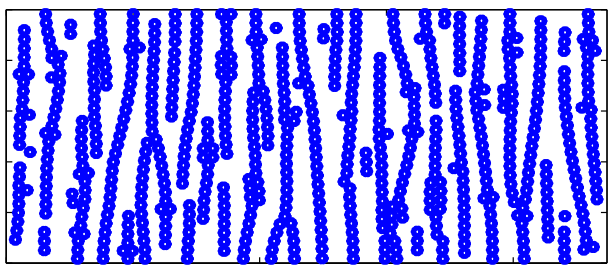

Time $=25.51$ milliseconds
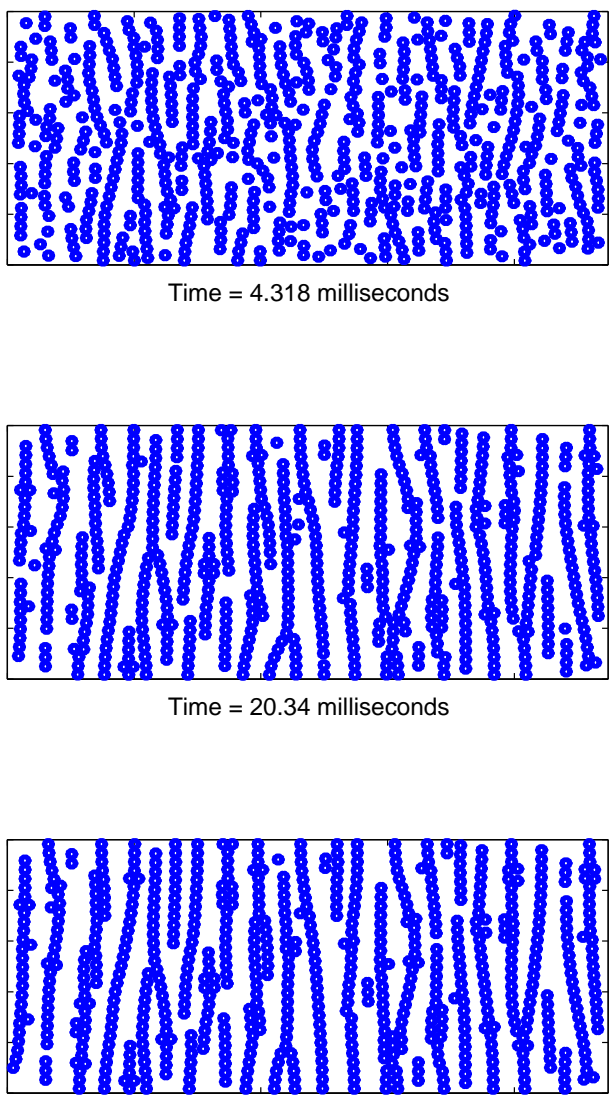

Time $=30.31$ milliseconds

Figure 2: dynamic simulations for MR fluid with $20 \%$ volume fraction.

dipoles within a viscous fluid; this results in (Jolly et. al [1997])

$$
t_{1} \cong \frac{C_{1} \eta_{c}}{\mu_{k} H_{0}^{2}} \varphi^{-n}+C_{2}
$$

with $n=5 / 3$. In this subsection, we undertake a numerical study of these time scales. Following the experimental procedure, we first derive values for such constants from effective permeability calculations. Finally, we estimate similar constants from a different macroscopic measurements, namely that of the "average kinetic energy."

We begin by examining the evolution of the effective permeability of the MR fluid as a function of time. As we said, we do so with the expectation that the effective permeability reflects the microstructure state within the MR fluid. The definition and the formulation for the effective permeability, which is based on theory of homogenization (Jikov [1994]), are derived in Ly et.al. [1998]. The effective permeability is defined as a matrix

$$
\mu_{\mathrm{eff}}=\left[\begin{array}{ll}
\bar{\mu}_{11} & \bar{\mu}_{12} \\
\bar{\mu}_{21} & \bar{\mu}_{22}
\end{array}\right]
$$




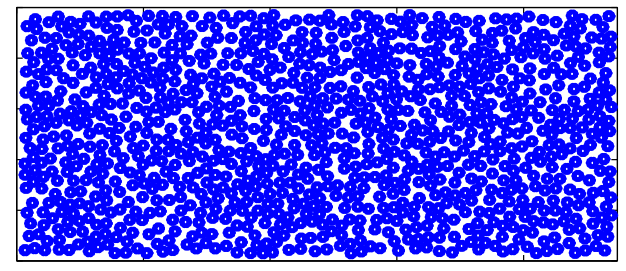

Time $=0$ milliseconds

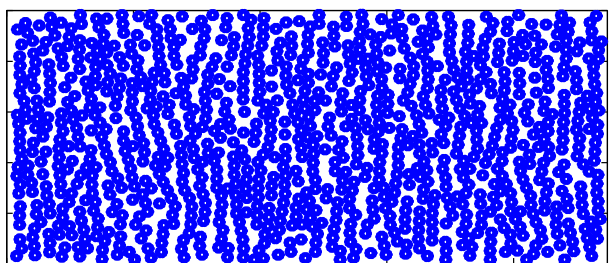

Time $=1.691$ milliseconds

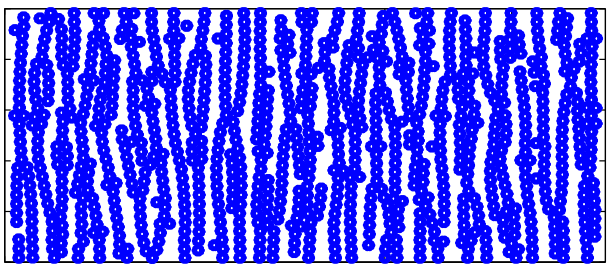

Time $=13.65$ milliseconds

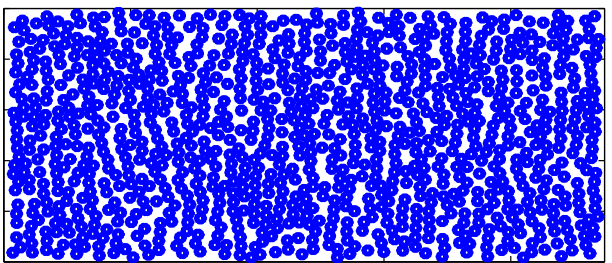

Time $=1.138$ milliseconds

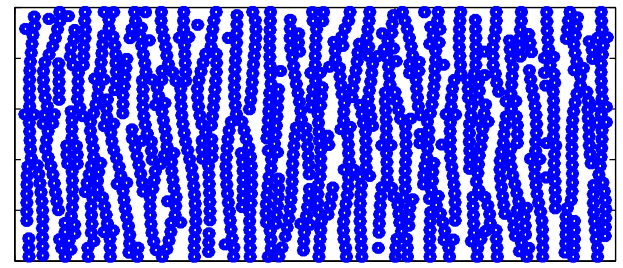

Time $=10.87$ milliseconds

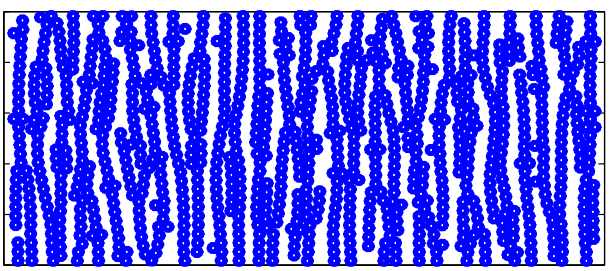

Time $=15.52$ milliseconds

Figure 3: dynamic simulations for MR fluid with 30\% volume fraction.

where

$$
\mu_{\mathrm{eff}}<\nabla \phi(\overrightarrow{\mathbf{x}})>=<\mu(\overrightarrow{\mathbf{x}}) \nabla \phi(\overrightarrow{\mathbf{x}})>,
$$

and $\langle\cdot\rangle$ denotes the spatial average. Using Green's identity and the boundary conditions (4.1), equation (4.3) yields explicitly

$$
\bar{\mu}_{12}=\frac{1}{H_{0} L_{x} L_{y}} \sum_{k=1}^{M}\left(\mu_{k}-\mu_{0}\right) \int_{\partial \Omega_{k}} \phi n_{x} d S
$$

and

$$
\bar{\mu}_{22}=\mu_{0}+\frac{1}{H_{0} L_{x} L_{y}} \sum_{k=1}^{M}\left(\mu_{k}-\mu_{0}\right) \int_{\partial \Omega_{k}} \phi n_{y} d S
$$

Because the applied magnetic field is in the vertical direction, $\bar{\mu}_{22}$ is a more relevant quantity and we denote by $\bar{\mu}:=\bar{\mu}_{22}$ the effective permeability reflecting the overall magnetic response of the MR fluid. In Figure 4, we display the effective permeability as a function of time. We remark that the effective permeabilities obtained from the dynamic simulations are roughly 
40-50\% smaller than those obtained from numerical and physical experiments in Simon et. al [1998]. One reason we suggest pertains to the percolation limit of particle separation. As pointed out in the study of Simon et. al [1998], the inter-particle distance plays a major role in the effective permeability in MR fluids: the permeability increases as the inter-particle distance decreases. For the two-dimensional case, it is necessary to allow the particle gap to approach $1 \%$ of the particle radius in order to achieve values comparable to those of experimental results. In our simulations, the gaps between particles were constrained to at least $4 \%$ of the radius. One could allow the particles in our study to get closer to $1 \%$ of the radius by refining the boundary integral element which, of course, would lead to prolonged computing time.
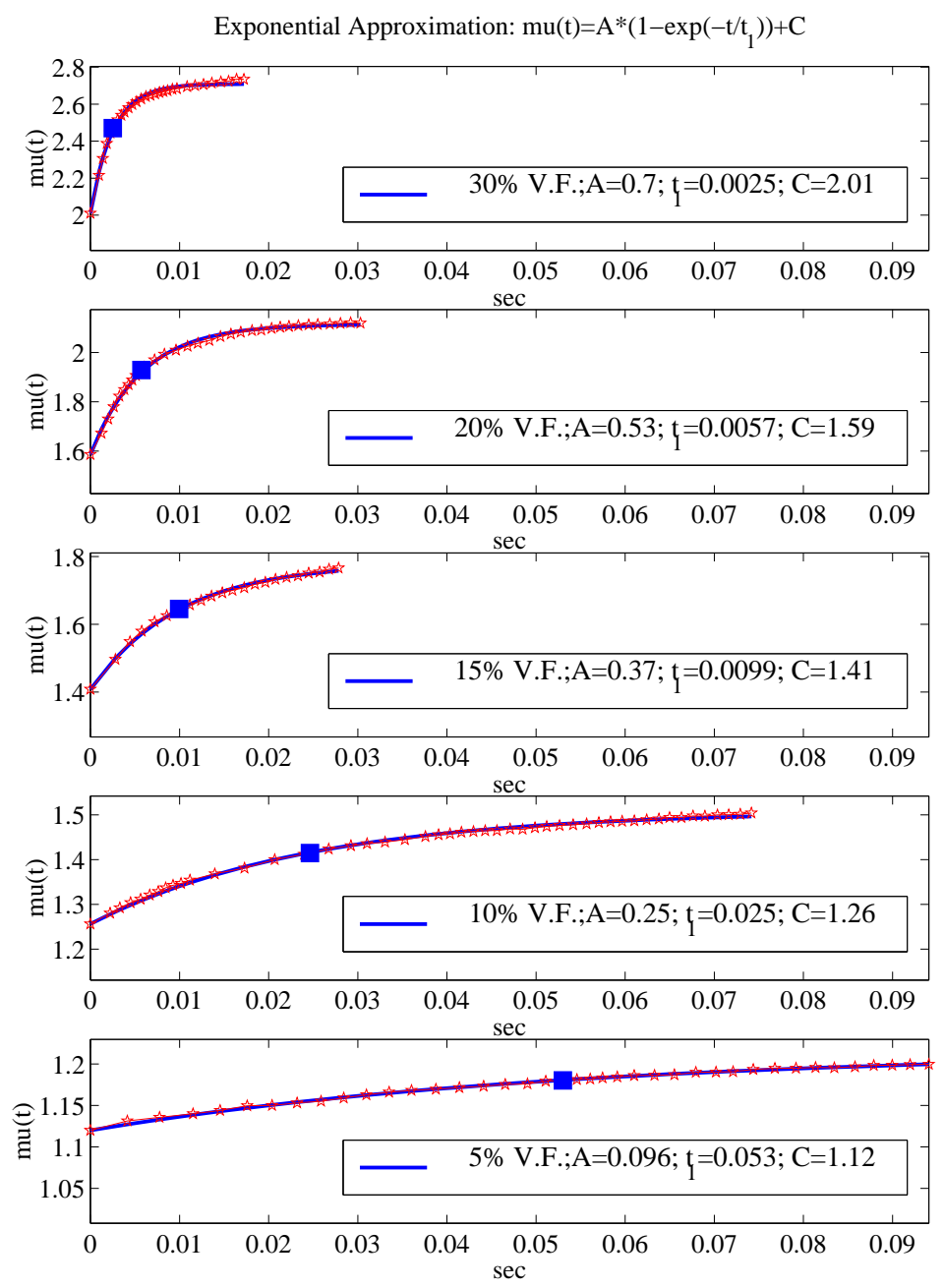

Figure 4: Least square fitting (line) on the computed effective permeability (star) and the time scale $t_{1}$ (solid square)

Figure 4 shows that the values of the effective permeabilities elevate faster for samples with higher concentration of iron. To identify the time-scales that correspond to this behav- 


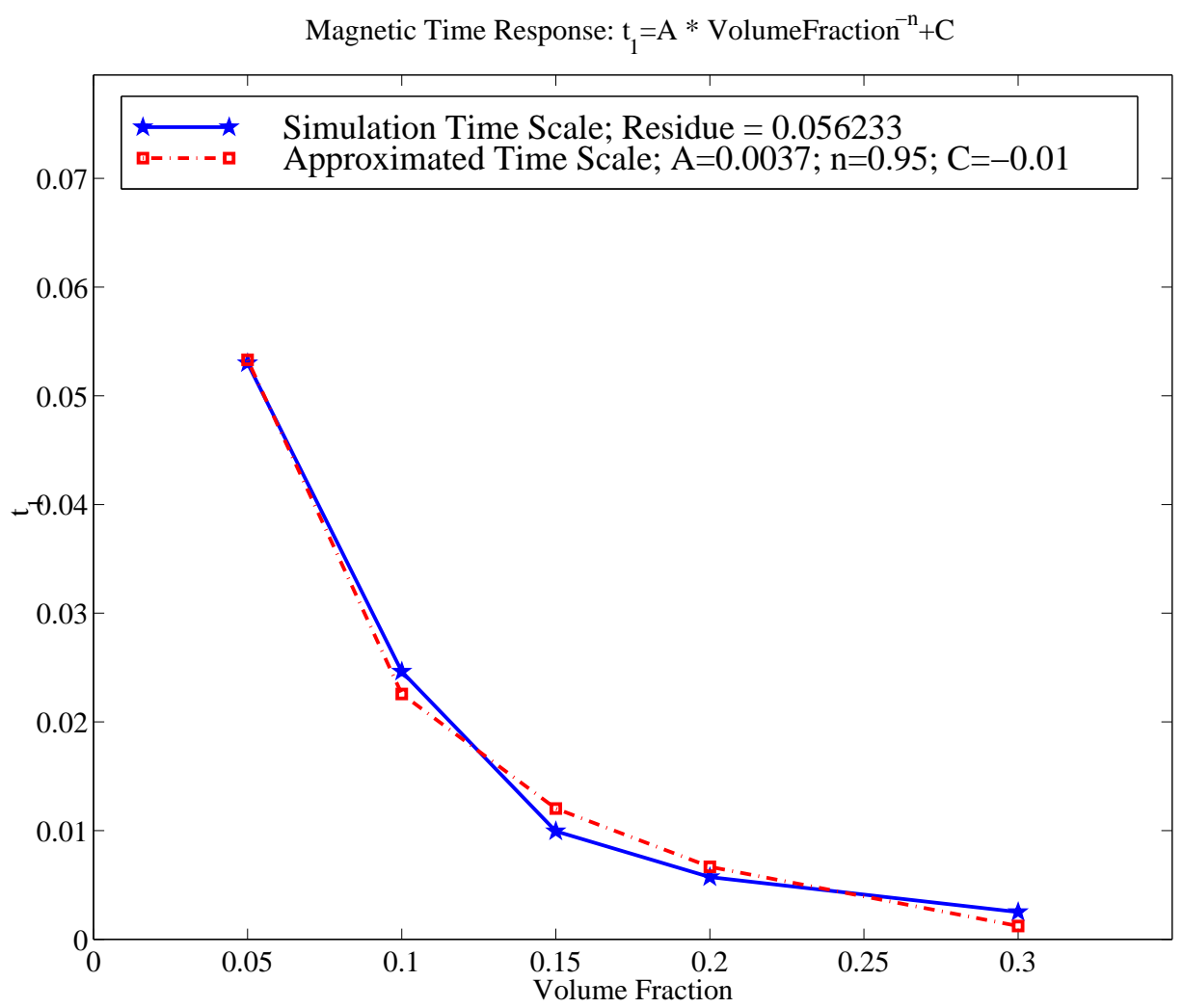

Figure 5: Time scale $t_{1}$ obtained from effective permeability and its least-square fitting for the power law (4.2)

ior, we perform an exponential fit to a function of the form $\bar{\mu}(t)=A\left(1-\exp \left(-\frac{t}{t_{1}}\right)\right)+C$ for each of the considered samples. The values of the time-scales $t_{1}$ for the respective volume fractions are displayed in Figure 4 by the large solid squares. To determine the dependence of the time scale $t_{1}$ on the volume fraction $\varphi$, we use equation (4.2) to fit the data in Figure 4 to find that $n \sim 1$ (see Figure 5). In this regard, our experiments indicate that the value of the power $n$ is rather insensitive to the initial configuration of the system.

Finally, motivated by the observation of initial rapid particle motion and subsequent slow rearrangement, we have monitored the evolution of the average particle kinetic energy $E(t)$ as another macroscopic measure of microstructure changes. For this we define $E(t)=$ $\frac{1}{2} \mathcal{M} \overline{\mathbf{V}}(t)^{2}$ where $\mathcal{M}$ is the particle mass and $\overline{\mathbf{V}}(t)$ is the average velocity,

$$
\overline{\mathbf{V}}(t)=\frac{1}{M} \sum_{k=1}^{M} \frac{\left|\overrightarrow{\mathbf{x}}_{k}(t)-\overrightarrow{\mathbf{x}}_{k}(t-\Delta t)\right|}{\Delta t} .
$$

Figure 6 displays the average particle kinetic energy $E(t)$ and confirms that $E(t)$, associated with the motion of the particles, decays at a rate that depends on the volume fraction. An exponential fit in time $E(t)=A \exp \left(-t / t_{1}\right)+C$ as in the previous case reveals that $\left\{t_{1}\right\}$ is approximately proportional to $\varphi^{-3.3 / 3}$. This is in remarkable agreement with the 

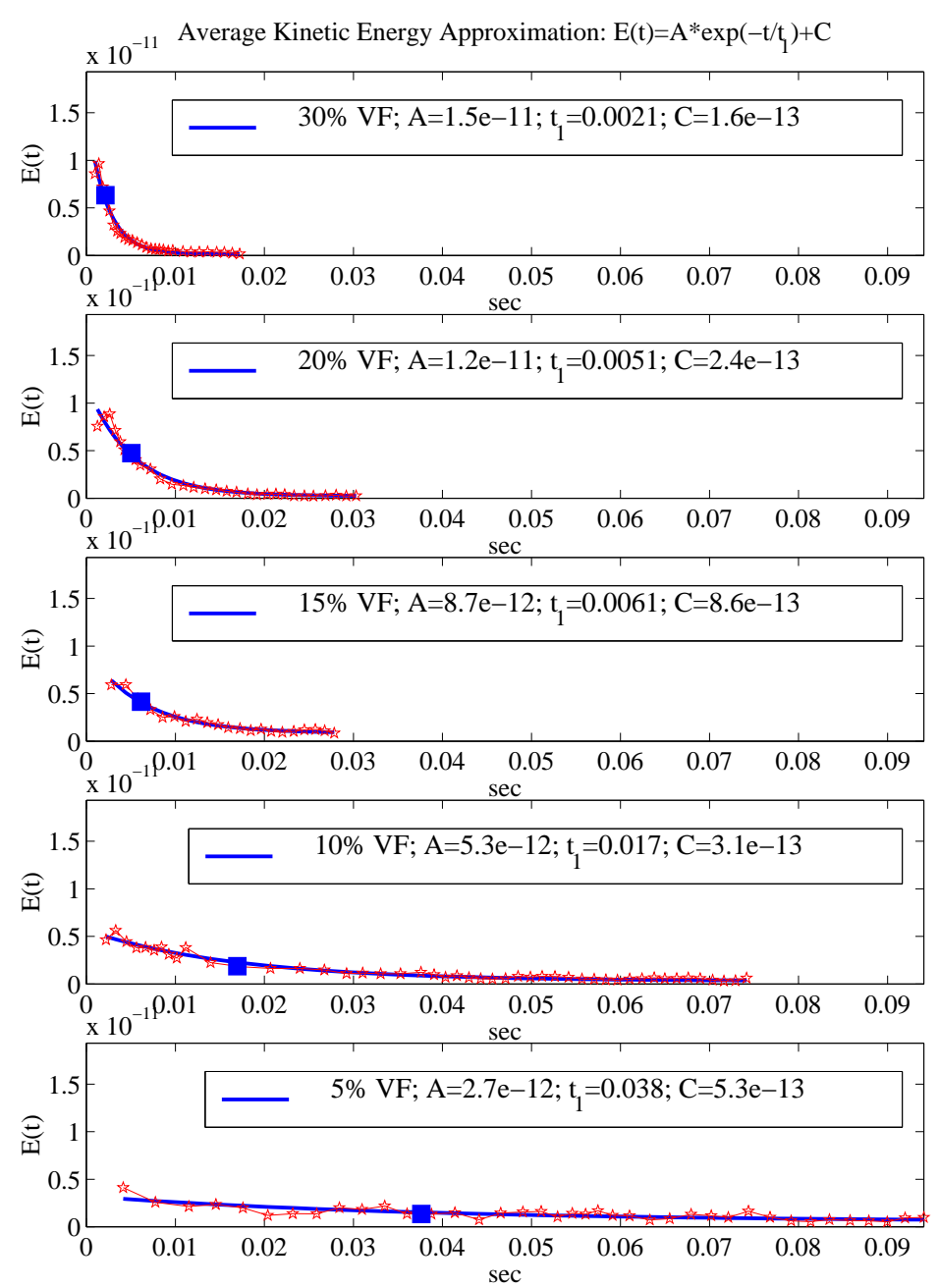

Figure 6: Numerical simulation kinetic energy $E(t)$ (star), its least-square approximation (line) and the time scale $t_{1}$ (solid square).

experimental measurements Blackwood et. al. [1994] and Jolly et. al. [1997] who have found $n$ in (4.2) to be between $2 / 3$ and $4 / 3$.

\section{Summary and Conclusions}

We have presented a computational technique to perform particle dynamics simulations of MR fluids upon application of an external magnetic field that, for the first time, fully account for all linear (long-range) magnetic interactions. To calculate these magnetic forces we solve a (highly oscillatory) magnetostatic problem at each instant in the evolution by appealing to a Fast Multipole Method on a boundary integral formulation. Additional hydrodynamic and repulsive forces are accounted for by Stokes drag and approximate hardsphere/hard-wall rejections, respectively. We have effectively used the resulting numerical 


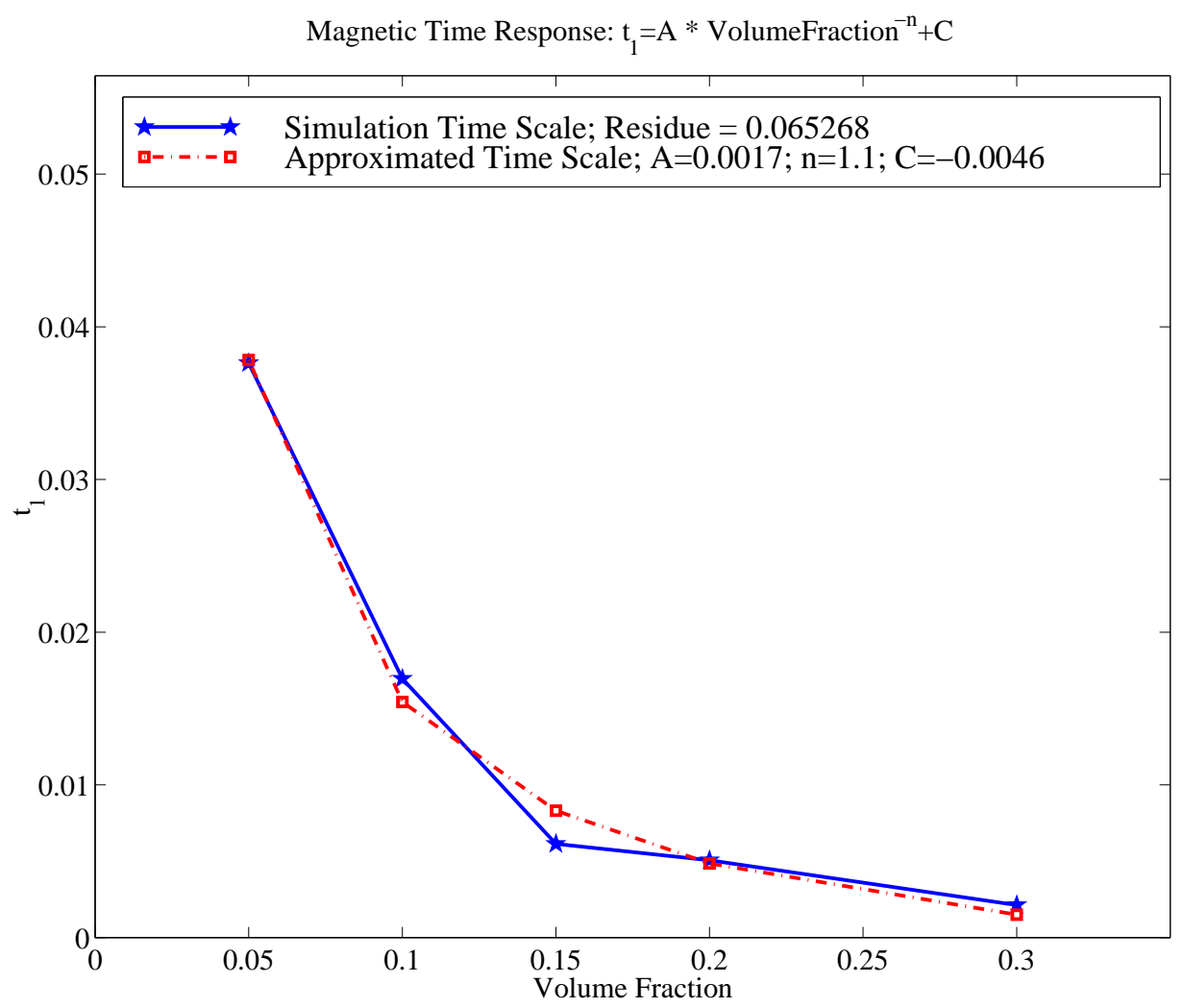

Figure 7: Time scale $t_{1}$ obtained from kinetic energy and its least-square fitting for the power law (4.2)

code to study a number of crucial experimental observables (effective permeability, response time scale) and have found our results in good agreement with experimental data.

\section{Acknowledgments}

This work was supported jointly by the National Science Foundation Industrial PostDoctoral Grant (No. DMS-9704963) and by the Lord Corporation through funds to the Center for Research in Scientific Computation at North Carolina State University. The research in this project was done when Hung V. Ly was a National Science Foundation Industrial Post-Doctoral Fellow at the Center for Research in Scientific Computation- North Carolina State University. F. Reitich gratefully acknowledges support from AFOSR through grant No. F49620-95-1-0113 and from NSF through grant No. DMS-9622555. H.T. Banks was supported in part by AFOSR under grants F49620-95-1-0236 and F49620-98-1-0180. 


\section{References}

[1] G.K. Batchelor, An Introduction to Fluid Dynamics, Cambridge, (1967).

[2] K.M. Blackwood, H. Block, P. Rattray, G. Tsangaris and D.N. Vorobiev, "The polarization, structuring and rheology of ER fluids," in Proceedings of the International Conference on Electro-Rheological Fluids (R. Tao, eds), World Scientific, Singapore (1994) pp. 3-21.

[3] R.T. Bonnecaze and J.F. Brady, "Dynamic simulation of an electrorheological fluid," Journal of Chemical Physics, 96 (1992), N3:2183-2202

[4] W.A. Bullough, Proceedings of the 5th International Conference on Electro-Rheological Fluids, Magneto-Rheological Suspensions and Associated Technology (W.A. Bullough, eds), World Scientific 1996.

[5] J.D. Carlson \& T.G. Duclos, "ER fluid clutches and brakes: Fluid property and mechanical design considerations," Electrorheological fluids, (J.D. Carlson, A.F. Sprecher and H. Conrad, eds), Technomics, Lancaster (1990), pp. 353-367

[6] J.D. Carlson \& K.D. Weiss, "A growing attraction to magnetic fluids," Machine Design, AUGUST 8 (1994) pp. 61-66.

[7] D.L. Colton, R. Kress, Integral Equation Methods in Scattering Theory, New York : Wiley, (1983).

[8] N. Engheta, W.D. Murphy, V. Rokhlin and M.S. Vassiliou, "The fast multipole method (FMM) for electromagnetic scattering problems," IEEE Transactions on Antennas and Propagation, 40 (1992), N6:634-642.

[9] L. Greengard and V. Rokhlin, "A fast algorithm for particle simulations," Journal of Computational Physics, 73 (1987), N2:325-348.

[10] L. Greengard, "The rapid evaluation of potential fields in particle systems," Cambridge: MIT Press (1988).

[11] L. Greengard and V. Rokhlin, "On the evaluation of electrostatic interactions in molecular modeling," Chemica Scripta, 29a (1989), Sep:139-144.

[12] L. Greengard and W.D. Gropp, "A parallel version of the fast multipole method," Computers \& Mathematics with Applications, 20 (1990a), N7:63-71.

[13] L. Greengard and J. Strain, "A fast algorithm for the evaluation of heat potentials," Communications on Pure and Applied Mathematics, 43 (1990b), N8:949-963.

[14] L. Greengard and M. Moura, "On the numerical evaluation of electrostatic fields in composite materials," Acta numerica, (1994), pp. 379-410.

[15] L. Greengard and M. C. Kropinski, "An integral equation approach to the incompressible Navier-Stokes equations in two dimensions," SIAM J. Sci. Comput., 20 (1999), N1:318-336. 
[16] K. C. Hass, "Computer simulations of nonequilibrium structure in electrorheological fluids," Physical Review E 47 (1993), N5:3362-3373.

[17] S. Hess and T. Weider, "Flow properties and structure changes in magnetorheological fluids via nonequilibrium molecular dynamics," Proc. XIIth International Congress on Rheology Quebec City, Canada (1996), pp. 313-314.

[18] M.A. Jaswon, Integral equation methods in potential theory and elastostatics, Academic Press, London, (1977).

[19] V. V. Jikov, S. M. Kozlov, and O. A. Oleinik, Homogenization of Differential Operators and Integral Functionals, Springer-Verlag, New York, (1994).

[20] M.R. Jolly, J.W. Bender \& R.T. Mathers, "Indirect measurements of microstructure development in magnetorheological fluids," International Conf. on Electrorheological Fluids and Magnetorheological Suspensions and their Applications, Yonezawa, Japan, July 22-25 (1997).

[21] M.R. Jolly, J.W. Bender \& J.D. Carlson, "Properties and applications of commercial magnetorheological fluids," Proc. of Smart Structures \& Materials, (SPIE), San Diego, California, March 2-3 (1998a) pp. 262-275.

[22] M.R. Jolly \& M. Nakano, "Properties and applications of commercial controllable fluids," Proc. of the 6th Int. Conf. on New Actuators, June 17-19 (1998b) pp. 411.

[23] P. Jones, J.A. Ma, and V. Rokhlin, "A fast direct algorithm for the solution of the Laplace equation on regions with fractal boundaries," Journal of Computational Physics, 113 (1994), N1:35-51.

[24] D. J. Klingenberg, F.van Swol, C. F. Zukoski, "Dynamic simulation of electrorheological suspensions," Journal of Chemical Physics 91 (1989), N12:7888-7895.

[25] R. Kress, Linear Integral Equations, Springer-Verlag, Berlin Heidelberg (1989).

[26] W. Leventon, "Fluid damper may make truck seats safer," Design News, Oct. 4 (1993) 185-186.

[27] H. V. Ly, F. Reitich, M. Jolly, H. T. Banks, and K. Ito, "Parameter Studies for the Magnetorheological Fluids Using the Fast Multipole Method," in preparation, (1998).

[28] A. McKenney, L. Greengard and A. Mayo, "A fast Poisson solver for complex geometries," Journal of Computational Physics, 118 (1995), N2:348-355.

[29] M. Mohebi, N. Jamasbi, and J. Liu, "Simulation of the formation of nonequilibrium structures in magnetorheological fluids subject to an external magnetic field," Physical Review E 54 (1996), N5:5407-5413.

[30] W.D. Murphy, V. Rokhlin and M.S. Vassiliou, "Acceleration methods for the iterative solution of electromagnetic scattering problems," Radio Science, 28 (1993), N1:1-12. 
[31] M. Parthasarathy and D. J. Klingenberg, "Electrorheology: mechanicsms and models," Material Science and Engineering R17 (1996), pp. 57-103.

[32] J. Rabinow, "The magnetic fluid clutch," AIEE Trans. 67 (1948), 1308-1315.

[33] V. Rokhlin "Rapid solution of integral equations of classical potential theory," Journal of Computational Physics, 60 (1985) 187-207.

[34] V. Rokhlin, "Rapid solution of integral equations of scattering theory in 2 dimensions," Journal of Computational Physics, 86 (1990), N2:414-439.

[35] T. Simon, F. Reitich, M. R. Jolly, K. Ito, and H. T. Banks, "Estimation of the effective permeability in magnetorheological fluids," CRSC TR-98-35, North Carolina State University, (1998); Journal of Intelligent Material Systems and Structures (submitted).

[36] R. Tao, Proceedings of the International Conference on Electro-Rheological Fluids (R. Tao, eds), World Scientific 1992.

[37] K.D. Weiss \& J.D. Carlson, "Macroscopic behavior of ER fluids: Technique for measuring response time," International Journal of Modern Physics B, 6 (1992) pp. 2609-2623.

[38] K.D. Weiss, T.G. Duclos, J.D. Carlson, M.J. Chrzan \& A.J. Margida, "High strength magneto- and electro-rheological fluids," Society of Automotive Engineers, SAE\#932451 (1993a).

[39] K.D. Weiss, J.D. Carlson \& J. Coulter, "Review: material aspects of electrorheological systems," Journal of Intelligent Material Systems and Structures, 4 (1993b) pp. 13-34.

[40] W.M. Winslow, "Method and means for translating electrical impulses into mechanical force," U.S. Patent 2,417,850 (1947).

[41] W.M. Winslow, "Induced fibration of suspensions," Journal of Applied Physics, 20 (1949), pp. 1137-1140. 